\title{
Nonequilibrium Dynamics and Magnetoviscosity of Moderately Concentrated Magnetic Liquids: A dynamic Mean-field Study
}

\author{
Patrick Ilg and Siegfried Hess \\ Institut für Theoretische Physik, Technische Universität Berlin, \\ Hardenbergstr. 36, D-10623 Berlin, Germany \\ Reprint requests to Dr. P. Ilg, E-mail: ilg@ physik.tu-berlin.de \\ Z. Naturforsch. 58a, 589-600 (2003); received September 20, 2003
}

\begin{abstract}
A mean-field Fokker-Planck equation approach to the dynamics of ferrofluids in the presence of a magnetic field and velocity gradients is proposed that incorporates magnetic dipole-dipole interactions of the colloidal particles. The model allows to study the combined effect of a magnetic field and dipolar interactions on the viscosity of the ferrofluid. It is found that dipolar interactions lead to additional non-Newtonian contributions to the stress tensor, which modify the behavior of the non-interacting system. The predictions of the present model are in qualitative agreement with experimental results, such as the enhancement as well as the different anisotropy of the magnetoviscous effect and the dependence on the symmetric velocity gradient.
\end{abstract}

Key words: Magnetohydrodynamics and Electrohydrodynamics; Magnetic Liquids; Rotational Flow and Vorticity; Suspensions, Dispersions, pastes, slurries, colloids; Kinetic Theory. PACS: 47.65.+a; 75.50.Mm; 47.32.-y; 83.80.Hj; 05.20.Dd.

\section{Introduction}

The so-called magnetoviscous effect in ferrofluids the dependence of the rheological behavior of colloidal suspensions of nano-sized ferromagnetic particles in a carrier liquid on external magnetic fields has attracted quite some attention in the recent literature, both from a scientific and an application point of view [1-4]. In dilute ferrofluids, the magnetoviscous effect is successfully explained by the hindrance of rotation of individual, non-interacting magnetic dipoles [5]. Experiments on commercial ferrofluids have revealed a quantitative and qualitative different behavior compared to the dilute regime, such as an order of magnitude increase of the magnetoviscous effect, a dependence not only on the local vorticity but also on the local strain rate of the flow, shear thinning behavior and the occurrence of normal stresses $[3,6]$. The failure of the kinetic model [5] to describe these phenomena is generally attributed to the neglect of magnetic dipolar interactions of the colloidal particles. Since dipolar interactions are long-ranged, they become important at concentrations as low as a few percent which are easily attained in commercial ferrofluids. The present contribution provides an extension of the non-interacting model to moderately concentrated ferrofluids with weak dipolar interactions.
A general statistical theory of magnetic fluids that covers dilute as well as concentrated suspensions with arbitrary strength of dipolar interactions is not available at present. For strong dipolar interactions the formation of chain-like aggregates is expected. A corresponding kinetic model has been proposed in [7]. In many ferrofluids, the strength of the dipolar interactions is comparable to the thermal energy [3], such that permanent aggregation is not expected. We here propose an extension of the kinetic model [5], that does not assume the existence of permanent chainlike aggregates but that incorporates magnetic dipole-dipole and excluded volume interactions in a mean-field approximation. The model is expected to apply in the dilute and semi-dilute, weakly interacting regime. Several results are obtained: A correction to the Langevin function describing the equilibrium magnetization of non-interacting magnetic dipoles is obtained, in agreement with results of [8]. The additional contributions of dipolar interactions to the hydrodynamic stress tensor are worked out. Contrary to the case of noninteracting magnetic dipoles, the stress tensor now depends also on the symmetric part of the velocity gradient. In case of uniaxial symmetry, the hydrodynamic stress tensor is of the same form as in the EricksenLeslie theory of nematic liquid crystals. The predictions of the present model agree qualitatively with ex- 
perimental results and address the shortcomings of the non-interacting model [5], such as the modified concentration dependence and anisotropy of the magnetoviscosity. Some quantitative comparison to the experimental results of [9] are offered also. It should be mentioned that a similar approach has been proposed already in [10], where, however, no dependence on the rate-of-strain tensor is considered, while such a dependence has been found experimentally in [11]. In addition, we keep higher order contributions in the strength of dipolar interactions compared to [10].

This paper is organized as follows: In Sect. 2, the kinetic model of semi-dilute ferrofluids is developed for equilibrium conditions. It is shown that the equilibrium behavior of this model agrees with the results of [8]. In Sect. 3, the kinetic model is extended to describe the dynamics of ferrofluids in the presence of an external flow field. The kinetic model is supplemented by the definition of the hydrodynamic stress tensor. The dynamical and rheological behavior of the present model is studied in Section 4. In case of uniaxial symmetry, the hydrodynamic stress tensor is found to be of the general form proposed in the Ericksen-Leslie theory of nematic liquid crystals. Explicit expressions for the viscosity coefficients are obtained. We also derive a closed magnetization equation within the so-called effective field approximation. The magnetization equation is a special case of the general equation proposed in [12] within a thermodynamic framework. Finally, some conclusions are drawn in Section 5.

\section{Model Definition and Equilibrium Properties}

Consider a system of $N$ interacting, spherical colloidal particles in a volume $V$. All particles are assumed to be identical, ferromagnetic monodomain particles of diameter $d$. Let $\boldsymbol{x}_{i}=\left\{\boldsymbol{r}^{(i)}, \boldsymbol{u}^{(i)}\right\}$ denote the five-dimensional vector describing the position $\boldsymbol{r}^{(i)}$ and orientation $\boldsymbol{u}^{(i)}$ of particle $i$, where $\boldsymbol{u}^{(i)}$ is a vector on the three-dimensional unit sphere. The particles are assumed to carry a permanent magnetic moment $\boldsymbol{\mu}^{(i)}=$ $\mu \boldsymbol{u}^{(i)}$. The total interaction potential may be written as

$$
U=-\mu \sum_{i=1}^{N} \boldsymbol{u}^{(i)} \cdot \boldsymbol{H}_{\mathrm{loc}}+\sum_{i<j} w_{i j}^{\mathrm{s}}+\sum_{i<j} w_{i j}^{\mathrm{dd}}
$$

Here and in the sequel, we use the following notation for dot products: $\boldsymbol{a} \cdot \boldsymbol{b} \equiv \sum_{\mu} a_{\mu} b_{\mu}$ and $\boldsymbol{A}: \boldsymbol{B} \equiv$ $\sum_{\mu \nu} A_{\mu \nu} B_{\nu \mu}$. The first term denotes the potential energy of an ideal paramagnetic gas in the local magnetic field $\boldsymbol{H}_{\text {loc. }}$. The second term is the potential energy of the non-magnetic system, where $w_{i j}^{\mathrm{s}}=w^{\mathrm{s}}\left(r_{i j}\right)$, with $\boldsymbol{r}_{i j}=\boldsymbol{r}^{(i)}-\boldsymbol{r}^{(j)}, r_{i j}^{2}=\boldsymbol{r}_{i j}^{2}$, is a spherical symmetric, short range, repulsive potential. In particular, we consider the case of hard spheres, $w^{\mathrm{s}}(r)=\infty$ if $r<d$ and zero otherwise, and soft spheres, $w^{\mathrm{s}}(r)=(r / d)^{-12}$. The dimensionless energy of two magnetic dipoles is described by

$$
\beta w_{i j}^{\mathrm{dd}}\left(\boldsymbol{r}_{i j}, \boldsymbol{u}^{(i)}, \boldsymbol{u}^{(j)}\right)=-3 \lambda\left(d / r_{i j}\right)^{3} \boldsymbol{u}^{(i)} \cdot \overline{\hat{\boldsymbol{r}}_{i j} \hat{\boldsymbol{r}}_{i j}} \cdot \boldsymbol{u}^{(j)},
$$

where $\beta=\left(k_{\mathrm{B}} T\right)^{-1}, k_{\mathrm{B}}$ and $T$ are Boltzmann's constant and temperature, respectively, $\boldsymbol{r}_{i j}=r_{i j} \hat{\boldsymbol{r}}_{i j}$, $\boldsymbol{a}=\left(\boldsymbol{a}+\boldsymbol{a}^{T}\right) / 2-(\operatorname{tr} \boldsymbol{a}) \mathbf{1} / 3$ denotes the symmetric traceless part of the matrix $\boldsymbol{a}$ and $\mathbf{1}$ is the threedimensional unit matrix. The dimensionless interaction parameter

$$
\lambda=\frac{\mu^{2}}{4 \pi \mu_{0} k_{\mathrm{B}} T d^{3}}
$$

is given by the ratio of the magnetic dipole-dipole energy of two colloidal particles of diameter $d$ in contact, over the thermal energy. It is well-known that due to the long range nature of the dipolar interactions, the magnetic properties of the system depend on the geometry of the container. In order to deal with this situation, we follow [8] and introduce a virtual cut-off radius $R_{\mathrm{c}}$ of the dipolar interactions. The effect of the dipole $j$ on dipole $i$ with $r_{i j}>R_{\mathrm{c}}$ is treated in a continuum approximation. Within the Weiss model, the resulting far-field contribution leads to a local magnetic field $\boldsymbol{H}_{\text {loc }}$ which is given by

$$
\boldsymbol{H}_{\mathrm{loc}}=\boldsymbol{H}+\frac{1}{3} \boldsymbol{M}
$$

where $\boldsymbol{M}$ denotes the magnetization of the sample. Finally, the virtual cut-off is removed, $R_{\mathrm{c}} \rightarrow \infty$. For further details see [8].

Exact results for the thermodynamic properties of the model system (1) are not available. Since the typical volume fraction of magnetic material in ferrofluids is low, the free energy of the system is conveniently expressed by the virial expansion. Let $f(\boldsymbol{u})$ denote the one-particle distribution function of finding the orientation $\boldsymbol{u}$ of an individual colloidal particle. The normalization is chosen such that $\int \mathrm{d}^{2} u f(\boldsymbol{u})=1$, where integration over the three-dimensional unit sphere is 
denoted by $\int \mathrm{d}^{2} u$. The free energy functional per particle of the system, $F[f]$, may be split into an ideal, $F_{0}[f]$, and an excess part, $F_{\text {ex }}[f]$. For the ideal system we choose an ideal gas of noninteracting magnetic dipoles,

$$
\begin{gathered}
F_{0}[f]=k_{\mathrm{B}} T\left[\ln n-1-\int \mathrm{d}^{2} u f(\boldsymbol{u}) \boldsymbol{u} \cdot \boldsymbol{h}_{\mathrm{loc}}\right. \\
\left.+\int \mathrm{d}^{2} u f(\boldsymbol{u}) \ln f(\boldsymbol{u})\right]
\end{gathered}
$$

where $n=N / V$ denotes the number density and $\boldsymbol{h}_{\text {loc }}=\mu \boldsymbol{H}_{\text {loc }} / k_{\mathrm{B}} T$ the dimensionless local magnetic field. For low concentrations, the excess part may be written in terms of the virial expansion as [13]

$$
\begin{gathered}
F_{\mathrm{ex}}[f]=-\frac{1}{2} n k_{\mathrm{B}} T \int \mathrm{d}^{2} u \int \mathrm{d}^{2} u^{\prime} f(\boldsymbol{u}) f\left(\boldsymbol{u}^{\prime}\right) b_{2}\left(\boldsymbol{u}, \boldsymbol{u}^{\prime}\right) \\
+\mathcal{O}\left(n^{2}\right) .
\end{gathered}
$$

The function $b_{2}$ is defined by

$$
b_{2}\left(\boldsymbol{u}, \boldsymbol{u}^{\prime}\right)=\int \mathrm{d}^{3} r\left(e^{-\beta w_{12}^{\mathrm{dd}}\left(\boldsymbol{r}, \boldsymbol{u}, \boldsymbol{u}^{\prime}\right)}-1\right) g_{\mathrm{sp}}(\boldsymbol{r})
$$

where $g_{\text {sp }}$ denotes the pair correlation function of the reference system $\left(w_{12}^{\mathrm{dd}}=0\right)$. The function $b_{2}$ can be interpreted as the change of the second virial coefficient due to the dipolar interactions. The integration over the three-dimensional spherical volume is denoted by $\int \mathrm{d}^{3} r$.

The function $b_{2}$, (7), can be expressed as a power series in the interaction parameter $\lambda$. Using the fact that the pair correlation function $g_{\mathrm{sp}}(r)$ is spherical symmetric, we arrive at

$$
\begin{aligned}
& b_{2}\left(\boldsymbol{u}, \boldsymbol{u}^{\prime}\right)=24 v \sum_{k=2}^{\infty} \lambda^{k} \frac{3^{k-1}}{(k-1) k !} c_{2, k} \\
& \int \frac{\mathrm{d}^{2} \hat{r}}{4 \pi}\left(\boldsymbol{u} \cdot \hat{\hat{\boldsymbol{r}} \hat{\boldsymbol{r}}} \cdot \boldsymbol{u}^{\prime}\right)^{k},
\end{aligned}
$$

where $v=\pi \mathrm{d}^{3} / 6$ denotes the hydrodynamic volume of the colloidal particles. The numerical coefficients

$$
c_{2, k}=3(k-1) \int_{0}^{\infty} \mathrm{d} x x^{2-3 k} g_{\mathrm{sp}}(x)
$$

depend on the particular choice of the short range potential $w^{\mathrm{s}}$ and the corresponding pair correlation function. Due to the spherical integration volume the term $k=1$ is missing in the sum of (8). In the low density limit, $g_{\mathrm{sp}}(x)$ can be approximated by $g_{\mathrm{sp}}(x) \approx$ $\exp \left(-\beta w_{\mathrm{s}}\right)$. If we consider the case of hard spheres, $g_{\mathrm{sp}}(x)$ can thus be identified in the low density limit with the Heaviside step function at $x=r / d=1$. In this case we have $c_{2, k}=1$. For power law repulsions, $\beta w_{\mathrm{s}}(x)=x^{-\nu}$, the coefficients $c_{2, k}$ are given by $c_{2, k}=\bar{k} \Gamma(\bar{k})$, where $\bar{k}=3(k-1) / \nu$. The socalled soft sphere potential is recovered for $\nu=12$. In this case, the coefficients $c_{2, k}$ are close to 1 for $k \leq 5$ and therefore similar to the value of the hard sphere system.

Inserting the expansion (8) into (6) one obtains

$F[f]=F_{0}[f]-\phi k_{\mathrm{B}} T \sum_{k=2}^{\infty} \lambda^{k} c_{2, k} G_{k}[f]+\mathcal{O}\left(\phi^{2}\right)$,

where $\phi=n v$ is the hydrodynamic volume fraction. We have found explicit expressions of the functionals $G_{k}$ for $k<5$,

$$
G_{2}[f]=\frac{2}{5}\left(\langle\overrightarrow{\boldsymbol{u u}}\rangle:\langle\overrightarrow{\boldsymbol{u u}}\rangle+\frac{10}{3}\right),
$$

$$
\begin{gathered}
G_{3}[f]=-\frac{2}{105}\left(\left\langle\overline{u_{\alpha} u_{\beta} u_{\gamma}}\right\rangle\right. \\
\left.\cdot\left\langle\overline{u_{\alpha} u_{\beta} u_{\gamma}}\right\rangle-\frac{42}{5}\langle\boldsymbol{u}\rangle \cdot\langle\boldsymbol{u}\rangle\right), \\
G_{4}[f]=\frac{1}{210}\left(\left\langle\overline{u_{\alpha} u_{\beta} u_{\gamma} u_{\delta}}\right\rangle\left\langle\overline{u_{\alpha} u_{\beta} u_{\gamma} u_{\delta}}\right\rangle\right. \\
\left.+\frac{48}{7}\langle\overline{\boldsymbol{u u}}\rangle:\langle\overline{\boldsymbol{u u}}\rangle+\frac{56}{5}\right) .
\end{gathered}
$$

Angular averages of arbitrary functions $a(\boldsymbol{u})$ with respect to the distribution function $f$ are denoted by

$$
\langle a\rangle=\int \mathrm{d}^{2} u a(\boldsymbol{u}) f(\boldsymbol{u}) .
$$

Note, that functionals $G_{k}[f]$ depend on the distribution function only via moments of $f$ up to order $k$.

The equilibrium distribution $f_{\text {eq }}$ is found by minimizing the functional (10) subject to the constraint of fixed normalization, $\int \mathrm{d}^{2} u f_{\text {eq }}(\boldsymbol{u})=1$. The result reads $f_{\text {eq }}(\boldsymbol{u})=\exp \left[-\beta V^{\mathrm{MF}}\left(\boldsymbol{u} ; f_{\text {eq }}\right)\right] / Z_{\text {eq }}$, where $Z_{\text {eq }}$ denotes the normalization constant. The mean-field potential is

$$
\beta V^{\mathrm{MF}}(\boldsymbol{u} ; f)=-\boldsymbol{u} \cdot \boldsymbol{h}_{\mathrm{loc}}-\phi \sum_{k=2}^{\infty} \lambda^{k} c_{2, k} \frac{\delta G_{k}[f]}{\delta f(\boldsymbol{u})} .
$$


Note that, due to the occurrence of moments in (15), the equilibrium distribution $f_{\text {eq }}$ has to be determined self-consistently signaling the mean-field character of the present model. Linearization in the volume fraction $\phi$ leads to

$$
\begin{aligned}
f_{\mathrm{eq}}(\boldsymbol{u})=f_{\alpha_{s}}(\boldsymbol{u})[1 & +\frac{8}{15} \lambda^{2} \phi c_{2,2} L_{2}\left(\alpha_{s}\right)\left\{P_{2}(\boldsymbol{u} \cdot \hat{\boldsymbol{H}})-L_{2}\left(\alpha_{s}\right)\right\} \\
& -\frac{8}{525} \lambda^{3} \phi c_{2,3}\left(L_{3}\left(\alpha_{s}\right)\left\{P_{3}(\boldsymbol{u} \cdot \hat{\boldsymbol{H}})-L_{3}\left(\alpha_{s}\right)\right\}-21 L_{1}\left(\alpha_{s}\right)\left\{\boldsymbol{u} \cdot \hat{\boldsymbol{H}}-L_{1}\left(\alpha_{s}\right)\right\}\right) \\
& +\frac{8}{3675} \lambda^{4} \phi c_{2,4}\left(L_{4}\left(\alpha_{s}\right)\left\{P_{4}(\boldsymbol{u} \cdot \hat{\boldsymbol{H}})-L_{4}\left(\alpha_{s}\right)\right\}+20 L_{2}\left(\alpha_{s}\right)\left\{P_{2}(\boldsymbol{u} \cdot \hat{\boldsymbol{H}})-L_{2}\left(\alpha_{s}\right)\right\}\right) \\
& \left.+\mathcal{O}\left(\lambda^{5} \phi\right)+\mathcal{O}\left(\lambda^{2} \phi^{2}\right)\right] .
\end{aligned}
$$

In (16) we have used functions $L_{j}$ which are defined recursively by $L_{j+1}(x)=L_{j-1}(x)-(2 j+1) L_{j}(x) / x$ with $L_{0}(x)=1$ and the Langevin function $L_{1}(x)=$ $\operatorname{coth}(x)-x^{-1}$.

The functions

$$
f_{\alpha}(\boldsymbol{u})=\frac{\alpha}{4 \pi \sinh (\alpha)} e^{\alpha \boldsymbol{u} \cdot \hat{\boldsymbol{H}}}
$$

are the equilibrium distribution functions in the absence of dipolar interactions. In (17) we have introduced the Langevin parameter $\alpha$ by $\boldsymbol{h}=\mu \boldsymbol{H} / k_{\mathrm{B}} T=$ $\alpha \hat{\boldsymbol{H}}$ with $\hat{\boldsymbol{H}}$ the unit vector in the direction of the magnetic field. Thus, $\alpha$ is a measure for the strength of the magnetic field relative to the thermal energy. The macroscopic magnetization is expressed as $\boldsymbol{M}=$ $M_{\text {sat }}\langle\boldsymbol{u}\rangle$. Thus, the local field $\boldsymbol{h}_{\text {loc }}$ can be expressed as $\boldsymbol{h}_{\text {loc }}=\alpha_{s} \hat{\boldsymbol{H}}$, with the effective Langevin parameter $\alpha_{s}=\alpha+\chi_{\mathrm{L}} L_{1}\left(\alpha_{s}\right)$. The Langevin susceptibility is defined by $\chi_{\mathrm{L}}=8 \phi \lambda$.

Evaluating the free energy functional (10) with the equilibrium distribution $f_{\text {eq }}$ one obtains the equilibrium free energy $F(\alpha)=F\left[f_{\text {eq }}\right]$ up to linear order in $\phi$,

$F\left(\alpha_{s}\right) / k_{\mathrm{B}} T=\ln \left(\frac{\alpha_{s}}{\sinh \left(\alpha_{s}\right)}\right)-\phi \sum_{k=2}^{\infty} \lambda^{k} c_{2, k} G_{k}\left(\alpha_{s}\right)$.

The functions $G_{k}\left(\alpha_{s}\right)$ are defined by $G_{k}\left(\alpha_{s}\right)=$ $G_{k}\left[f_{\text {eq }}\right]$. Explicit expressions for the first functions $G_{k}$, obtained from (11)-(13) combined with (16), are given in the appendix. We have confirmed that (18) and (A1) - (A3) agree with the results of [8] in the case of hard spheres where $c_{2, k}=1$. The advantage of the present formulation compared to the results of [8] is, that (A1) - (A3) simplify the discussion of the properties and asymptotic behavior of the functions $G_{i}(x)$.

We define equilibrium order parameters by $S_{j}^{\text {eq }} \equiv$ $\left\langle P_{j}(\boldsymbol{u} \cdot \hat{\boldsymbol{H}})\right\rangle_{\text {eq }}$, where $P_{j}$ are Legendre polynomials of degree $j$. The function $S^{\text {eq }}$ can be obtained by multiplying (16) by $\boldsymbol{u} \cdot \hat{\boldsymbol{H}}$ and subsequent integration over $\boldsymbol{u}$, or from (18) by $S_{1}^{\mathrm{eq}}\left(\alpha_{s}\right)=d F\left(\alpha_{s}\right) / d \alpha_{s}$. Linearization in the small quantity $\phi$ leads to the final result

$$
\begin{aligned}
S_{1}^{\mathrm{eq}}(\alpha)= & L_{1}(\alpha)+\chi_{\mathrm{L}} L_{1}(\alpha) L_{1}^{\prime}(\alpha) \\
& +\phi \sum_{k=2}^{\infty} c_{2, k} \lambda^{k} G_{k}^{\prime}(\alpha)
\end{aligned}
$$

where the prime denotes the total derivative. Equation (19) is identical with (4.24a) of [8] for $c_{2, k}=1$. Figure 1 shows a comparison of (19) with $c_{2, k}=1$ for $k \leq 4$ and $c_{2, k}=0$ for $k>4$ to the results of the molecular dynamics simulations of [14]. In [14], the volume fraction was chosen as $\phi=0.157$, and the dipolar interaction was $\lambda=1$, which is rather large for the present study. From Fig. 1 one notices that appreciable corrections of the Langevin magnetization occur for intermediate values of $\alpha$. For the parameters considered, the simulation results are in remarkable agreement with (19) if truncated at $k=4$. In the regime of strong dipolar couplings, $\lambda \gtrsim 2$, where corrections to the Langevin magnetization are stronger [14], the truncation of the expansion (19) is not admissible. This regime is left for future research. The truncation of the expansion at $k=2$ gives similar results to the truncation at $k=4$ for $\lambda \approx 1$. In Fig. 1 we include also the function $L_{1}\left(\alpha_{s}\right)$, which is obtained by neglecting all higher order corrections in $\lambda$ in (19) if the linearization in $\phi$ is not performed. As has been noted in [14], this 


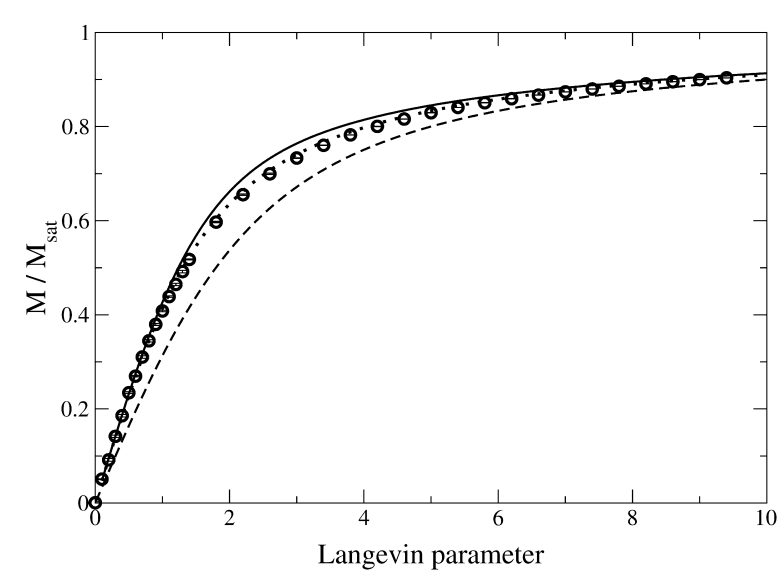

Fig. 1. Equilibrium magnetization as a function of the Langevin parameter for volume fraction $\phi=0.157$ and $\lambda=1$. Symbols are the result of molecular dynamics simulations presented in [14]. The dashed line is the Langevin function $L_{1}(\alpha)$, while the solid line corresponds to (19), where the infinite sum has been truncated at $k=4$. The dotted line is the result of the approximation $L_{1}\left(\alpha+\chi_{\mathrm{L}} L_{1}(\alpha)\right)$.

approximation describes their numerical data very well for the present choice of parameters. For later use, we give the expression for $S_{2}^{\text {eq }}$ in linear order in $\phi$ :

$$
\begin{aligned}
S_{2}^{\mathrm{eq}}(\alpha)= & L_{2}(\alpha)+\chi_{\mathrm{L}} L_{2}^{\prime}(\alpha) L_{1}(\alpha) \\
& +\phi \sum_{k=2}^{\infty} c_{2, k} \lambda^{k} J_{k}^{\prime}(\alpha)
\end{aligned}
$$

where

$$
\begin{aligned}
J_{2}^{\prime}(\alpha)= & \frac{8}{525} L_{2}(\alpha)\left[18 L_{4}(\alpha)\right. \\
& \left.+10 L_{2}(\alpha)+7-35 L_{2}(\alpha)^{2}\right] .
\end{aligned}
$$

\section{Mean-Field Kinetic Model}

The model introduced in Sect. 2 is now extended to describe the nonequilibrium dynamics of ferrofluids in the presence of an external flow field $\boldsymbol{v}(\boldsymbol{r})$. The oneparticle distribution function $f(\boldsymbol{u})$ now becomes timedependent, $f(\boldsymbol{u} ; t)$, and denotes the probability density of finding the orientation $\boldsymbol{u}$ of an individual colloidal particle at time $t$. For convenience, the explicit dependence of $f$ on $t$ is frequently suppressed in the sequel. The orientational dynamics of a ferromagnetic colloidal particle under the combined action of the local vorticity of the flow $\Omega=\frac{1}{2} \nabla \times \boldsymbol{v}$, Brownian mo- tion, and the action of the potential $V^{\text {eff }}$ is given by the kinetic equation $[2,5]$

$$
\partial_{t} f=-\mathcal{L} \cdot[\Omega f]+\mathcal{L} \cdot D_{\text {rot }}\left[\mathcal{L} f+f \mathcal{L}\left(\beta V^{\mathrm{eff}}\right)\right] .
$$

The rotational diffusion coefficient is $D_{\text {rot }}=$ $1 /\left(2 \tau_{\text {rot }}\right) . \tau_{\text {rot }}=3 \eta_{\mathrm{s}} v / k_{\mathrm{B}} T$ denotes the rotational relaxation time. The rotational operator is $\mathcal{L}=\boldsymbol{u} \times \partial / \partial \boldsymbol{u}$ with $\partial / \partial \boldsymbol{u}$ the gradient on the unit sphere. In the absence of flow, we assume that the effective potential $V^{\text {eff }}$ can be identified with the static mean-field potential $V^{\mathrm{MF}}$, (15). A similar approach was proposed by one of the authors in [15] in order to describe the dynamics of nematic liquid crystals. For simple fluids such an approach has been proposed and tested recently in [16].

In the presence of a symmetric velocity gradient $\boldsymbol{D}=\nabla \boldsymbol{v}$, an additional contribution to the effective potential $V^{\text {eff }}$ of the kinetic equation (22) arises. In the case of non-spherical particles, this contribution leads to the so-called flow alignment phenomenon $[15,17]$. In the present case, the additional contribution is due to flow-induced structures that can be formed even in a hard sphere system. The distortion of the pair correlation function due to shear flow has been studied experimentally [18] and theoretically [19]. For small distortions, the pair correlation function $g(\boldsymbol{r} ; t)$ satisfies the time evolution equation [19]

$$
\partial_{t} g+\boldsymbol{r} \cdot\left(\nabla_{\boldsymbol{r}} \boldsymbol{v}\right) \cdot \nabla_{\boldsymbol{r}} g+\frac{1}{\tau}\left(g-g_{\mathrm{sp}}\right)=0,
$$

where $\tau$ denotes a translational relaxation time. The stationary solution to (23) is given by

$$
g(\boldsymbol{r})=g_{\mathrm{sp}}(r)-\tau \boldsymbol{D}: \hat{\boldsymbol{r}} \hat{\boldsymbol{r}} r g_{\mathrm{sp}}^{\prime}(r) .
$$

Results of recent nonequilibrium molecular dynamics simulations of a dipolar model fluid confirm that (24) provides a reasonable description of the shear-induced distortion of the pair correlation function [20]. The distortion of the pair correlation function leads to an additional contribution to the effective potential which to lowest order in $\lambda$ reads

$$
\begin{aligned}
V^{\mathrm{D}}(\boldsymbol{u} ; f)= & n \int \mathrm{d}^{2} u^{\prime} f\left(\boldsymbol{u}^{\prime}\right) \\
& \cdot \int \mathrm{d}^{3} r w_{12}^{\mathrm{dd}}\left(\boldsymbol{r}, \boldsymbol{u}, \boldsymbol{u}^{\prime}\right)\left(g(\boldsymbol{r})-g_{\mathrm{sp}}(r)\right) .
\end{aligned}
$$

Equation (25) has an immediate interpretation as the flow-induced modification of the (self-consistently averaged) mean dipolar interaction potential. The effective potential $V^{\text {eff }}$ entering the kinetic equation (22) is 
obtained as $V^{\mathrm{eff}}=V^{\mathrm{MF}}+V^{\mathrm{D}}$. Inserting (24) into (25), the kinetic equation (22) takes to form

$$
\begin{aligned}
\partial_{t} f=-\mathcal{L} \cdot[ & \left\{\boldsymbol{\Omega}-\sigma_{0} \chi_{\mathrm{L}} \boldsymbol{u} \times \boldsymbol{D} \cdot\langle\boldsymbol{u}\rangle\right. \\
& \left.-D_{\mathrm{rot}} \mathcal{L}\left(\beta V^{\mathrm{MF}}\right) f\right]+D_{\mathrm{rot}} \mathcal{L}^{2} f,
\end{aligned}
$$

where $\sigma_{0}=3 \tau /\left(5 \tau_{\text {rot }}\right)$. In (26), we have assumed $g_{\mathrm{sp}}(r) \rightarrow 1$ for $r \rightarrow \infty$ and $g_{\mathrm{sp}}(0)=0$ due to excluded volume interactions. The kinetic equation for non-interacting dipoles, [5], is recovered from (26) in the limit $\lambda \rightarrow 0$. The use of (24) for the flow-induced distortion of the pair correlation function limits the validity of (26) to weak flows. More precisely, we expect (24) to apply for $\tau|\boldsymbol{D}| \lesssim 1$. In addition, the backreaction of the orientational on the translational dynamics is neglected. Since in (25) we have kept only the lowest order term in $\lambda$, further considerations are restricted to the regime of weak dipolar interactions $\lambda \ll 1$. In this regime, the mean-field potential (15) simplifies to

$\beta V^{\mathrm{MF}}(\boldsymbol{u} ; f)=-\boldsymbol{u} \cdot \boldsymbol{h}_{\mathrm{loc}}-\frac{4}{5} \lambda^{2} \phi c_{2,2} \overline{\boldsymbol{u u}}:\langle\overline{\boldsymbol{u u}}\rangle$.

As has been noted in Sect. 2, the truncation (27) of the mean-field potential provides a good approximation for the equilibrium magnetization even for $\chi_{\mathrm{L}} \approx 1$, $\lambda \approx 1$.

It is interesting to compare the hydrodynamic drag appearing in (22) to the corresponding terms in case of ellipsoidal-shaped particles with axis ratio $r$. For the latter, the contribution of $\boldsymbol{D}$ to the hydrodynamic drag is quadratic in $\boldsymbol{u}$ and proportional to the so-called shape factor $B=\left(r^{2}-1\right) /\left(r^{2}+1\right)$ as well as to the rotational relaxation time $\tau_{\text {rot }}$ [17]. Spherical particles correspond to $r=1$ and $B=0$. If it would be allowed to replace $\langle\boldsymbol{u}\rangle$ in the second term on the right hand side of (26) by $\boldsymbol{u}$, the flow contribution of the present model would be identical to a dilute solution of ferromagnetic ellipsoidal particles with shape factor $B=-\sigma_{0} \chi_{\mathrm{L}}$. A negative value of $B$ is characteristic of an oblate ellipsoid. This finding has an intuitive interpretation, since dipolar interactions favor a head-to-tail arrangement in contrast to side-side configurations. Thus, the particles effectively appear extended in the direction perpendicular to the magnetic moment. In Sect. 4 the validity and limitations of the analogy are discussed for special quantities.

For irrotational flows $\Omega=\mathbf{0}$, the stationary solution to the kinetic equation (22) reads $f_{\text {st }}=$ $\exp \left(-\beta V^{\text {eff }}\right) / Z_{\text {st }}$, where $Z_{\text {st }}$ denotes the normalization constant. Note that $f_{\text {st }}$ is formally identical to the equilibrium distribution $f_{\text {eq }}$, if the magnetic field $\boldsymbol{h}_{\text {loc }}$ is replaced by the effective field $\boldsymbol{h}_{\text {eff }}=\boldsymbol{h}_{\text {loc }}-$ $(6 / 5) \chi_{\mathrm{L}} \tau \boldsymbol{D} \cdot\langle\boldsymbol{u}\rangle$.

From the kinetic equation (22) a hierarchy of moment equations can be derived. Multiplying (22) by $\boldsymbol{u}$ and integration over $\boldsymbol{u}$ leads to

$$
\begin{aligned}
\partial_{t}\langle\boldsymbol{u}\rangle= & \boldsymbol{\Omega} \times\langle\boldsymbol{u}\rangle+\sigma_{0} \chi_{\mathrm{L}}(\langle\boldsymbol{u} \boldsymbol{u}\rangle \cdot \boldsymbol{D} \cdot\langle\boldsymbol{u}\rangle-\boldsymbol{D} \cdot\langle\boldsymbol{u}\rangle) \\
& +D_{\operatorname{rot}}(\boldsymbol{h}-\langle\boldsymbol{u} \boldsymbol{u}\rangle \cdot \boldsymbol{h})+D_{\operatorname{rot}} \chi_{\mathrm{L}}(\langle\boldsymbol{u}\rangle-\langle\boldsymbol{u} \boldsymbol{u}\rangle \cdot\langle\boldsymbol{u}\rangle) \\
& +\frac{1}{5} c_{2,2} D_{\operatorname{rot}} \chi_{\mathrm{L}} \lambda(\langle\overline{\boldsymbol{u} u}\rangle \cdot\langle\boldsymbol{u}\rangle-\langle\boldsymbol{u} \boldsymbol{u} \boldsymbol{u}\rangle:\langle\overline{\boldsymbol{u} u}\rangle) \\
& -2 D_{\operatorname{rot}}\langle\boldsymbol{u}\rangle .
\end{aligned}
$$

Analogously, higher order moment equations are derived. In order to study the magnetoviscous effect, the kinetic equation (22) has to be supplemented by the expression for the hydrodynamic stress tensor $\boldsymbol{T}$. Considering only rotational degrees of freedom, the stress tensor is antisymmetric and has the form $\boldsymbol{T}^{\text {rot }}=$ $3 \eta_{\mathrm{s}} \phi \boldsymbol{\epsilon} \cdot\left(\boldsymbol{\Omega}-\left\langle\boldsymbol{\omega}^{\mathrm{p}}\right\rangle\right)$, where $\boldsymbol{\epsilon}$ is the conventional total antisymmetric (Levi-Civita) tensor of rank three. The average angular velocity $\left\langle\boldsymbol{\omega}^{\mathrm{p}}\right\rangle$ of the colloidal particles can be inferred from the kinetic equation (22), which can be cast into the form $\partial_{t} f=-\mathcal{L} \cdot\left[\boldsymbol{\omega}^{\mathrm{p}} f\right]$. Thus, $\boldsymbol{T}^{\text {rot }}$ is given by $2 \boldsymbol{T}^{\text {rot }}=n \boldsymbol{\epsilon} \cdot\left\langle\mathcal{L} V^{\mathrm{eff}}\right\rangle$ and becomes upon inserting (25) and (27)

$$
\begin{aligned}
& \boldsymbol{T}^{\mathrm{rot}}=\frac{n k_{\mathrm{B}} T}{2}( \alpha[\hat{\boldsymbol{H}}\langle\boldsymbol{u}\rangle-\langle\boldsymbol{u}\rangle \hat{\boldsymbol{H}}]-\frac{6}{5} \tau \chi_{\mathrm{L}} \\
&\cdot[\boldsymbol{D} \cdot\langle\boldsymbol{u}\rangle\langle\boldsymbol{u}\rangle-\langle\boldsymbol{u}\rangle\langle\boldsymbol{u}\rangle \cdot \boldsymbol{D}]) .
\end{aligned}
$$

One has $\chi_{\mathrm{L}}=0$ in the non-interacting model [5], so that the hydrodynamic stress arises from hindered rotations of individual magnetic moments in the magnetic field. The configurational contribution to the hydrodynamic stress is given by

$$
\begin{array}{r}
\boldsymbol{T}^{\mathrm{conf}}=\frac{n^{2}}{2} \int \mathrm{d}^{3} r \int \mathrm{d} \boldsymbol{u} \int \mathrm{d} \boldsymbol{u}^{\prime} f(\boldsymbol{u}) f\left(\boldsymbol{u}^{\prime}\right) \\
\cdot g^{(2)}\left(\boldsymbol{r}, \boldsymbol{u}, \boldsymbol{u}^{\prime}\right) \boldsymbol{r} \nabla_{\boldsymbol{r}} \Phi_{12}\left(\boldsymbol{r}, \boldsymbol{u}, \boldsymbol{u}^{\prime}\right),
\end{array}
$$

where $\Phi_{12}\left(\boldsymbol{r}, \boldsymbol{u}, \boldsymbol{u}^{\prime}\right)=w_{\mathrm{sp}}(r)+w_{12}^{\mathrm{dd}}\left(\boldsymbol{r}, \boldsymbol{u}, \boldsymbol{u}^{\prime}\right)$ is the full two particle interaction potential and $g^{(2)}$ denotes the full pair correlation function. The distortion of $g^{(2)}$ from its equilibrium $g_{\mathrm{eq}}^{(2)}$ is assumed to be described 
also by (23), where $g$ is now replaced by $g^{(2)}$. In this case, the stationary solution reads to leading order

$$
\begin{aligned}
g^{(2)}\left(\boldsymbol{r}, \boldsymbol{u}, \boldsymbol{u}^{\prime}\right)= & g_{\mathrm{eq}}^{(2)}\left(\boldsymbol{r}, \boldsymbol{u}, \boldsymbol{u}^{\prime}\right) \\
& -\tau\left(\nabla_{\boldsymbol{r}} \boldsymbol{v}\right): \boldsymbol{r} \nabla_{\boldsymbol{r}} g_{\mathrm{eq}}^{(2)}\left(\boldsymbol{r}, \boldsymbol{u}, \boldsymbol{u}^{\prime}\right) .
\end{aligned}
$$

Note, that (31) reduces to (24) only if the (anisotropic) contribution of the dipolar potential to the pair correlation function is neglected.

Inserting (31) into (30), the deviation of $\boldsymbol{T}^{\text {conf }}$ from the equilibrium stress becomes

$$
\Delta \boldsymbol{T}_{\mu \nu}^{\mathrm{conf}}=\eta_{\mu \nu \alpha \beta}^{\mathrm{conf}} \nabla_{\alpha} v_{\beta}
$$

with the viscosity tensor

$$
\begin{aligned}
\eta_{\mu \nu \alpha \beta}^{\operatorname{conf}}= & \frac{n^{2} \tau}{2 k_{\mathrm{B}} T} \int \mathrm{d}^{3} r \int \mathrm{d} \boldsymbol{u} \int \mathrm{d} \boldsymbol{u}^{\prime} f(\boldsymbol{u}) f\left(\boldsymbol{u}^{\prime}\right) \\
& \cdot g_{\mathrm{eq}}^{(2)}\left(\boldsymbol{r}, \boldsymbol{u}, \boldsymbol{u}^{\prime}\right) r_{\mu}\left(\nabla_{\nu} \Phi_{12}\right) r_{\alpha}\left(\nabla_{\beta} \Phi_{12}\right) .
\end{aligned}
$$

From (33) we notice that the viscosity tensor $\boldsymbol{\eta}^{\text {conf }}$ obeys $\eta_{\mu \nu \alpha \beta}^{\text {conf }}=\eta_{\alpha \beta \mu \nu}^{\text {conf }}$ and is positive semi-definite, i.e. $\eta_{\mu \nu \alpha \beta}^{\text {conf }} a_{\mu \nu} a_{\alpha \beta} \geq 0$ for arbitrary second-rank tensors $\boldsymbol{a}$.

Inserting the spherical and the dipole-dipole interaction potential into (33) yields symmetric and antisymmetric contributions to the stress tensor, such that the total hydrodynamic stress tensor becomes

$$
\boldsymbol{T}=\boldsymbol{T}^{\mathrm{rot}}+\boldsymbol{T}^{\mathrm{conf}}=\boldsymbol{T}^{\mathrm{s}}+\boldsymbol{T}^{\mathrm{a}},
$$

with the symmetric part,

$$
\begin{aligned}
\boldsymbol{T}^{\mathrm{s}}= & \left(2 \eta_{0}-\frac{2}{3}\left(c_{1}-3\right) a\langle\boldsymbol{u}\rangle^{2}\right) \\
& \cdot \boldsymbol{D}-\frac{7}{2} a[\boldsymbol{W} \cdot\langle\boldsymbol{u}\rangle\langle\boldsymbol{u}\rangle-\langle\boldsymbol{u}\rangle\langle\boldsymbol{u}\rangle \cdot \boldsymbol{W}] \\
& +a\left(c_{1}-3\right)[\boldsymbol{D} \cdot\langle\boldsymbol{u}\rangle\langle\boldsymbol{u}\rangle+\langle\boldsymbol{u}\rangle\langle\boldsymbol{u}\rangle \cdot \boldsymbol{D}]
\end{aligned}
$$

and the antisymmetric part

$$
\boldsymbol{T}^{\mathrm{a}}=\frac{\alpha n k_{\mathrm{B}} T}{2}(\hat{\boldsymbol{H}}\langle\boldsymbol{u}\rangle-\langle\boldsymbol{u}\rangle \hat{\boldsymbol{H}}) .
$$

In (35) we have introduced $\boldsymbol{W}=\left[(\nabla \boldsymbol{v})^{T}-\nabla \boldsymbol{v}\right] / 2$ and the quantity $a=2 \eta_{\mathrm{s}} \sigma \lambda \phi^{2}$, where $\sigma=72 \tau /\left(35 \tau_{\text {rot }}\right)$. The shear viscosity of the isotropic suspension is

$$
\eta_{0}=\eta_{\mathrm{s}}\left(1+\frac{5}{2} \phi+b \phi^{2}\right),
$$

where $\eta_{\mathrm{s}}$ is the shear viscosity of the pure solvent, $b=\frac{7}{6} c_{4} \sigma$. Note, that the isotropic interactions alter the value of the Newtonian viscosity, while dipolar interactions lead to additional, non-Newtonian contributions to the stress tensor. Diagonal contributions to $\boldsymbol{T}^{\mathrm{s}}$ have been suppressed in (35), since they can be compensated by the scalar pressure. Note also that the stress tensor $\boldsymbol{T}$ is symmetric in the absence of an applied magnetic field. The quantities $c_{1}$ and $c_{4}$ result from the short range interaction contribution to the stress tensor and depend on the detailed form of the interaction potential,

$$
c_{k}=\int_{0}^{\infty} \mathrm{d} x x^{k}\left[\beta w_{\mathrm{s}}^{\prime}(x)\right]^{2} g_{\mathrm{sp}}(x),
$$

where prime denotes the total derivative and $x=r / d$. The coefficients $c_{k}$ are non-negative, $c_{k} \geq 0$. Evaluation of the coefficients $c_{1}$ and $c_{4}$ for the case of hard spheres suffers from the discontinuity of the potential. For power law repulsion, $\beta w_{\mathrm{S}}(x)=x^{-\nu}$, the integration in (38) can be done analytically in the low density regime, giving $c_{k}=\nu \Gamma((2 \nu+1-k) / \nu)$. For soft spheres, $\nu=12$, we find $c_{1}=12$ and $c_{4} \approx 11.0$. We mention that the $\phi^{2}$ contribution to $\eta_{0}$ in (37) stems from the non-magnetic interactions of the colloidal particles. This contribution is of the form $G_{\mathrm{s}} \tau$, where $G_{\mathrm{s}}$ is the Born-Green expression of the equilibrium shear modulus of a system of spherical particles interacting with the potential $w_{\mathrm{s}}$ [21].

Equations (26), (27), (34), (35) and (36) are our main result and constitute the mean-field kinetic model of the nonequilibrium dynamics of dilute, weakly interacting ferrofluids proposed here. The present model extends the work of [5] to the regime of weak dipolar interactions, $\lambda \ll 1$. If the dimensionless dipolar interaction parameter $\lambda$ goes to zero, the model of non-interacting magnetic dipoles proposed in [5] is recovered with a renormalized zero-field viscosity $\eta_{0}$. The present model contains the additional parameter $\sigma$, which is a measure for the ratio of translational and rotational relaxation times. Simple estimates of the translational and rotational relaxation times lead to $\sigma \approx 6\left(r_{0} / d\right)^{2}$, where $r_{0}$ is a typical length scale associated with the formation of flow-induced structures. If $r_{0}$ is identified with the typical distance between colloidal particles, the parameter $\sigma$ can be estimated as $\sigma \approx 2 \phi^{-2 / 3}$. On the other hand, if $r_{0}$ is identified with the diameter $d$ of the colloidal particles, one obtains $\sigma=6$. These estimates of $\sigma$ agree with each other for $\phi \approx 0.2$, which is slightly above the expected range of validity of the present model. Alternatively, if one 


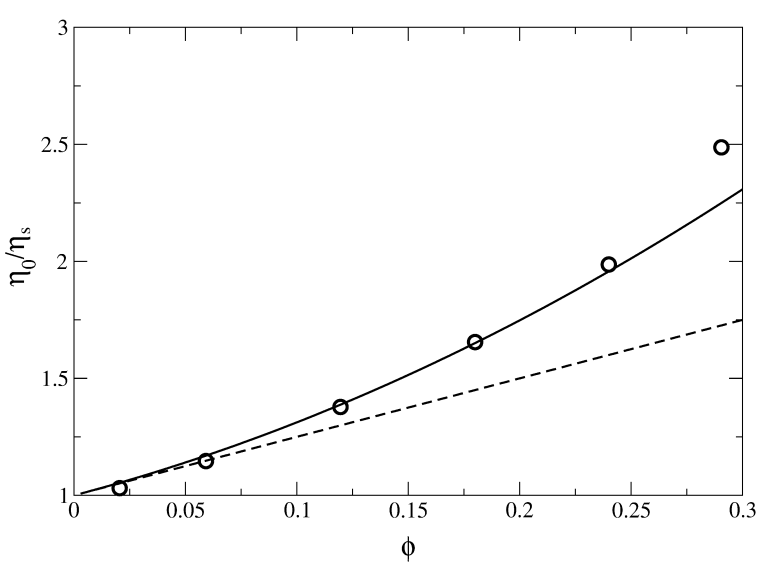

Fig. 2. Shear viscosity $\eta_{0} / \eta_{\mathrm{s}}$ of the isotropic suspension $(\boldsymbol{H}=0)$ as a function of the volume fraction $\phi$. The dashed an full lines are the theoretical predictions $\eta_{0} / \eta_{\mathrm{s}}=1+$ $2.5 \phi+b \phi^{2}$ with $b=0$ (Einstein) and $b=6.2$ (Batchelor), respectively.

requires the expression (37) for the zero-field viscosity to correspond to Batchelor's result, (37) with $b=6.2$ (see [3] and discussion therein), the parameter $\sigma$ is given by $\sigma=186 /\left(35 c_{4}\right)$, and thus related to the interaction potential $w_{\mathrm{s}}$. In case of soft spheres, agreement with Batchelor's result leads to $\sigma \approx 0.5$. In the sequel we consider $\sigma$ as parameter with $\sigma=\mathcal{O}(1)$. Figure 2 shows zero-field zero-shear viscosity $\eta_{0}$ of a kerosene based ferrofluid as a function of the volume fraction $\phi$. The data are taken from [3]. From Fig. 2 we notice that Batchelor's result describes the experimental data well for volume fractions $\phi \lesssim 0.25$.

\section{Results for Uniaxial Symmetry}

The stress tensor $\boldsymbol{T}$, $(35,36)$, depends explicitly on the first moment of the distribution function only. However, all the moments are coupled dynamically, as can be seen already from the first moment equation (28), such that the values of second and third moments are needed in order to determine the first moment and the stress tensor. Therefore, closed form equations for the dynamics of the stress tensor (34) in terms of low order moments necessarily introduce approximations to the underlying kinetic model. In a previous work [22], we have studied the assumption of uniaxial symmetry of the distribution function applied to the non-interacting kinetic model of [5]. Motivated by the good accuracy of the assumption of uniaxial symmetry for that model found in [22], we employ this assumption also for the present case.

In the uniaxial phase, the distribution function $f(\boldsymbol{u} ; t)$ is symmetric with respect to rotations around the director $\boldsymbol{n}, f(\boldsymbol{u} ; t)=f_{\text {uni }}(\boldsymbol{u} \cdot \boldsymbol{n} ; t)$, such that $f$ can be represented as

$$
f_{\text {uni }}(\boldsymbol{u} \cdot \boldsymbol{n} ; t)=\frac{1}{4 \pi} \sum_{j=0}^{\infty} \frac{1}{2 j+1} S_{j}(t) P_{j}(\boldsymbol{u} \cdot \boldsymbol{n}) .
$$

The scalar orientational order parameters $S_{j}$ are defined as $S_{j}=\left\langle P_{j}(\boldsymbol{u} \cdot \boldsymbol{n})\right\rangle$, where, as before, $P_{j}$ are Legendre polynomials. In case of uniaxial symmetry, the first moments take the form

$$
\begin{aligned}
\langle\boldsymbol{u}\rangle=S_{1} \boldsymbol{n}, & \langle\overrightarrow{\boldsymbol{u u}}\rangle=S_{2} \overrightarrow{\boldsymbol{n n}}, \\
\left\langle u_{\alpha} u_{\beta} u_{\gamma}\right\rangle= & S_{3} n_{\alpha} n_{\beta} n_{\gamma} \\
& +\frac{S_{1}-S_{3}}{5}\left(\delta_{\alpha \beta} n_{\gamma}+\delta_{\alpha \gamma} n_{\beta}+\delta_{\beta \gamma} n_{\alpha}\right) .
\end{aligned}
$$

For example, the distribution functions (16) and (17) are uniaxial symmetric with respect to the direction of the magnetic field, $\boldsymbol{n}=\hat{\boldsymbol{H}}$. Expressions for the equilibrium order parameters $S_{i}^{\text {eq }}$ are given in (19) and (20) for $i=1,2$.

Inserting Eqs. (40) into (28), the following time evolution equation for the orientational order parameter $S_{1}$ is obtained:

$$
\begin{aligned}
\dot{S}_{1}= & -\frac{2}{3} \sigma_{0} \chi_{\mathrm{L}} S_{1}\left(1-S_{2}\right)(\boldsymbol{D}: \boldsymbol{n n}) \\
& +\frac{2}{3} D_{\mathrm{rot}}\left(1-S_{2}\right) \boldsymbol{h}_{\mathrm{loc}} \cdot \boldsymbol{n}-2 D_{\mathrm{rot}} S_{1},
\end{aligned}
$$

with the local magnetic field $\boldsymbol{h}_{\text {loc }}=\boldsymbol{h}+\chi_{\mathrm{L}} S_{1} \boldsymbol{n}$. From (41), the angle between the direction of the magnetic field and the magnetization can be calculated:

$$
\begin{aligned}
\hat{\boldsymbol{H}} \cdot \boldsymbol{n}= & \frac{3 S_{1}^{2}}{h\left(1-S_{2}\right)}\left[1-\frac{1}{3} \chi_{\mathrm{L}}\left(1-S_{2}\right)\right. \\
& \left.+\frac{2}{3} \sigma_{0} \chi_{\mathrm{L}}\left(1-S_{2}\right) \tau_{\mathrm{rot}} \boldsymbol{D}: \boldsymbol{n n} \cdot\right]
\end{aligned}
$$

In equilibrium, where $S_{i}=S_{i}^{\mathrm{eq}}$, the magnetization is perfectly aligned with the magnetic field, $\hat{\boldsymbol{H}} \cdot \boldsymbol{n}=1$. From (40) and (28), also the balance equation for the director $\boldsymbol{n}$ is derived,

$$
(\boldsymbol{I}-\boldsymbol{n n}) \cdot\left[\boldsymbol{H}^{\mathrm{mol}}-\gamma_{1} \boldsymbol{N}-\gamma_{2} \boldsymbol{D} \cdot \boldsymbol{n}\right]=\mathbf{0},
$$




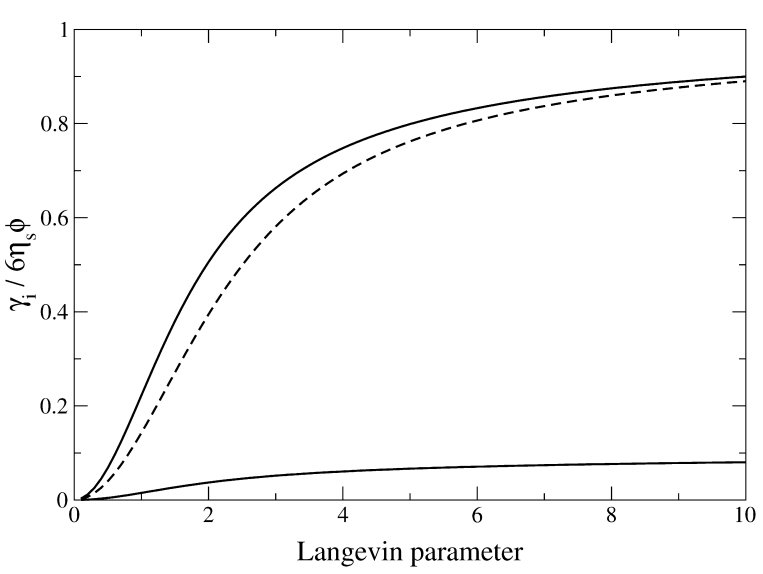

Fig. 3. Dimensionless viscosity coefficients $\gamma_{i} / 6 \eta_{\mathrm{s}} \phi$ defined in (44) as a function of the Langevin parameter $\alpha$. The upper solid line corresponds to $\gamma_{1}$, the lower solid line to $\gamma_{2}$. The dashed line is the result for $\gamma_{1}$ in the absence of dipolar interactions. In this case $\gamma_{2}=0$. Weak flow conditions are assumed so that the orientational order parameters are well approximated by their equilibrium values. The Langevin susceptibility was chosen as $\chi_{\mathrm{L}}=1$ and $\sigma=0.5$.

which is of the general form assumed in the EricksenLeslie theory of nematic liquid crystals [2]. $N=\dot{n}-$ $\boldsymbol{\Omega} \times \boldsymbol{n}$ is the corotational derivative of the director $\boldsymbol{n}$. Here, the molecular field $\boldsymbol{H}^{\mathrm{mol}}=n k_{\mathrm{B}} T S_{1} \boldsymbol{h}$ and the viscosity coefficients are given by

$$
\begin{aligned}
\gamma_{1} & =6 \eta_{\mathrm{s}} \phi \frac{3 S_{1}^{2}}{2+S_{2}} \\
\gamma_{2} & =\frac{7}{4} \eta_{\mathrm{s}} \phi \sigma \chi_{\mathrm{L}} S_{1}^{2} .
\end{aligned}
$$

The dependence of the viscosity coefficients $\gamma_{i}$ on the magnetic field is illustrated in Fig. 4 for weak flow conditions, where the orientational order parameters $S_{i}$ are well approximated by their equilibrium values $S_{i}^{\text {eq }}$. From Fig. 3 we observe that dipolar interactions increase the value of the viscosity coefficient $\gamma_{1}$ and lead to non-vanishing coefficient $\gamma_{2}$.

Inserting (40) into (35) and (36), and using (28), the hydrodynamic stress tensor is of the form assumed in the Ericksen-Leslie theory [2],

$$
\begin{aligned}
\boldsymbol{T}= & \alpha_{1}(\boldsymbol{D}: \boldsymbol{n n}) \boldsymbol{n n}+\alpha_{2} \boldsymbol{n N}+\alpha_{3} \boldsymbol{N n} \\
& +\alpha_{4} \boldsymbol{D}+\alpha_{5} \boldsymbol{n n} \cdot \boldsymbol{D}+\alpha_{6} \boldsymbol{D} \cdot \boldsymbol{n n},
\end{aligned}
$$

where the Leslie coefficients $\alpha_{i}$ are given by

$$
\begin{aligned}
\alpha_{1} & =0, \\
\alpha_{2} & =-3 \eta_{\mathrm{s}} \phi \frac{3 S_{1}^{2}}{2+S_{2}}+7 \eta_{\mathrm{s}} \sigma \lambda \phi^{2} S_{1}^{2}, \\
\alpha_{3} & =3 \eta_{\mathrm{s}} \phi \frac{3 S_{1}^{2}}{2+S_{2}}+7 \eta_{\mathrm{s}} \sigma \lambda \phi^{2} S_{1}^{2}, \\
\alpha_{4} & =2 \eta_{0}+4\left(1-c_{1} / 3\right) \eta_{\mathrm{s}} \sigma \lambda \phi^{2} S_{1}^{2}, \\
\alpha_{5} & =\left(c_{1}-13 / 2\right) 2 \eta_{\mathrm{s}} \sigma \lambda \phi^{2} S_{1}^{2}, \\
\alpha_{6} & =\left(c_{1}+1 / 2\right) 2 \eta_{\mathrm{s}} \sigma \lambda \phi^{2} S_{1}^{2} .
\end{aligned}
$$

The viscosity coefficients $\gamma_{i}$ are given in terms of $\alpha_{i}$ as $\gamma_{1}=\alpha_{3}-\alpha_{2}$ and $\gamma_{2}=\alpha_{6}-\alpha_{5}$. A relation between the Leslie coefficients can be derived from Onsager's reciprocity relation, $\alpha_{2}+\alpha_{3}=\alpha_{6}-\alpha_{5}$, which is known as Parodi's relation $[2,17]$. Parodi's relation is readily verified from (46) and (47). Note, that in the limit $\lambda \rightarrow 0$, the result of the noninteracting model of [22] is recovered from Eqs. (45), (46) and (47). From (46) and (47) we observe that dipolar interactions increase the number of independent viscosity coefficients compared to the noninteracting model.

\subsection{Effective Field Approximation}

In the so-called Effective Field Approximation (EFA) [5], a special family $f_{\xi_{e}}$ of uniaxial distribution functions is considered that is obtained by replacing the magnetic field $\boldsymbol{h}$ in (17) with an effective field $\boldsymbol{\xi}_{e}$. Motivated by the good accuracy of the EFA for the non-interacting model [5], we consider the EFA also for the present model.

In more general terms, the EFA can be interpreted as the Quasi-Equilibrium Approximation, where only the magnetization is kept as macroscopic variable (see e.g. [17]). Extremizing the free energy functional (10) subject to the constraint of fixed normalization and fixed value of the first moment yields the quasiequilibrium distribution $f_{\xi_{e}}$. Also in the present case, $f_{\xi_{e}}$ is obtained from the equilibrium distribution $f_{\text {eq }}$ if the magnetic field $\boldsymbol{h}$ in (16) is replaced by an effective field $\boldsymbol{\xi}_{e}$. Since the equilibrium distribution $f_{\text {eq }}$ is uniaxially symmetric around the magnetic field direction $\hat{\boldsymbol{H}}$, the distribution function $f_{\xi_{e}}$ is uniaxially symmetric with respect to the direction of the effective field $\boldsymbol{n}=\hat{\boldsymbol{\xi}}_{e}$, where $\boldsymbol{\xi}_{e}=\xi_{e} \hat{\boldsymbol{\xi}}_{e}$ and $\xi_{e}$ denotes the norm of $\boldsymbol{\xi}_{e}$. Consequently, the result of the previous section applies to the EFA. In particular, the moments $\langle\boldsymbol{u}\rangle$ and 
$\langle\boldsymbol{u} \boldsymbol{u}\rangle$ within the EFA are given by (40), where the scalar orientational order parameters are obtained from their equilibrium values by $S_{j}=S_{j}^{\mathrm{eq}}\left(\xi_{e}\right)$.

\section{Magnetization Equation}

From the moment equation (28) a closed equation for the magnetization $\boldsymbol{M}=M_{\text {sat }}\langle\boldsymbol{u}\rangle$ can be derived within the EFA which reads

$$
\begin{aligned}
\dot{\boldsymbol{M}}-\boldsymbol{\Omega} \times \boldsymbol{M}= & -\frac{1}{\nu_{1}} \boldsymbol{\Lambda}-\frac{1}{\nu_{2}} \boldsymbol{\Lambda} \cdot \boldsymbol{M} \boldsymbol{M} \\
& +\lambda_{2} \boldsymbol{D} \cdot \boldsymbol{M}+\lambda_{3} \boldsymbol{D}: \boldsymbol{M M} \boldsymbol{M},
\end{aligned}
$$

where $\boldsymbol{\Lambda}=k_{\mathrm{B}} T\left(\boldsymbol{\xi}_{e}-\boldsymbol{h}\right) / \mu$ is the (dimensional) deviation of the effective field from the magnetic field. The coefficients $\nu_{i}$ and $\lambda_{i}$ are defined as

$$
\begin{aligned}
& \frac{1}{\nu_{1}}=3 D_{\text {rot }} \chi_{\mathrm{L}} A\left(\xi_{e}\right), \frac{1}{\nu_{2}}=-\frac{\mu_{0}}{6 \eta_{\mathrm{s}} \phi} B\left(\xi_{e}\right), \\
& \lambda_{2}=-\sigma_{0} \chi_{\mathrm{L}} A\left(\xi_{e}\right), \lambda_{3}=\frac{\sigma_{0} \chi_{\mathrm{L}}}{M_{\text {sat }}^{2}} B\left(\xi_{e}\right) .
\end{aligned}
$$

The functions $A\left(\xi_{e}\right)$ and $B\left(\xi_{e}\right)$ are given by

$$
\begin{aligned}
A\left(\xi_{e}\right) \equiv & \frac{2+S_{2}\left(\xi_{e}\right)}{3} \\
= & 1-\frac{L_{1}\left(\xi_{e}\right)}{\xi_{e}}+\chi_{\mathrm{L}} \frac{L_{1}\left(\xi_{e}\right)}{\xi_{e}} \cdot\left(L_{1}\left(\xi_{e}\right)^{2}-L_{2}\left(\xi_{e}\right)\right) \\
& +\frac{1}{3} c_{2,2} \phi \lambda^{2} J_{2}^{\prime}\left(\xi_{e}\right),
\end{aligned}
$$

and $B\left(\xi_{e}\right) \equiv S_{2}\left(\xi_{e}\right) / S_{1}\left(\xi_{e}\right)^{2}$,

$$
\begin{aligned}
B\left(\xi_{e}\right)= & \frac{L_{2}\left(\xi_{e}\right)}{L_{1}\left(\xi_{e}\right)^{2}} \\
& +\chi_{\mathrm{L}}\left(1+L_{2}\left(\xi_{e}\right)\left[1-\frac{5+L_{2}\left(\xi_{e}\right)}{3 L_{1}\left(\xi_{e}\right)^{2}}\right]\right) \\
& +c_{2,2} \lambda \phi^{2} \frac{L_{1}\left(\xi_{e}\right) J_{2}^{\prime}\left(\xi_{e}\right)-2 L_{2}\left(\xi_{e}\right) G_{2}^{\prime}\left(\xi_{e}\right)}{L_{1}\left(\xi_{e}\right)^{2}} .
\end{aligned}
$$

The magnetization equation (48) is a special case of (15) of [12], which has been derived within a thermodynamic framework. The coefficients appearing in the magnetization equation, however, cannot be determined within the thermodynamic approach. For the special case $\lambda_{i}=0,(48)$ has been derived in [10] in linear order in $\lambda$ and $\phi$, within the EFA, starting from an $N$-particle Fokker-Planck equation. The expression for $A\left(\xi_{e}\right)$ given by (51) is identical to the result of [10] to first order in $\lambda$, while the result of [10] for the coefficient $B\left(\xi_{e}\right)$, coincides with (52) only for $\lambda=0$. However, correcting (15) of [10] for the missing factor $L_{1}\left(\xi_{e}\right) / \xi_{e}$ [23], also the results for the coefficient $B\left(\xi_{e}\right)$ agree with (52). In [17], the magnetization equation (48) has been derived within a kinetic model of non-interacting, ferromagnetic colloidal particles with an ellipsoidal shape. Comparing (50) to (77) of [17], we notice that the transport coefficients $\lambda_{i}$ are of a similar form in both cases. For weak fields, the coefficient $\lambda_{2}$ approaches a constant value $\lambda_{2}(0)=-\frac{2}{3} \sigma_{0} \chi_{\mathrm{L}}$. This result is identical to that of a non-interacting system of ellipsoidal particles with shape factor $B=-\frac{10}{9} \sigma_{0} \chi_{\mathrm{L}}$, see [17]. It should be mentioned that the coefficient $\lambda_{2}$ has recently been measured experimentally in [11] for a commercial ferrofluid. Since strong dipolar interactions occur in the fluid used in this experiment, the present model cannot be used to explain the experiment quantitatively.

\section{Relaxation Times}

Analytical results for the magnetization dynamics (48) can be obtained for small deviations from the equilibrium values. To linear order in $\boldsymbol{\Lambda}$ and in the absence of velocity gradients, (48) becomes

$$
\dot{\boldsymbol{M}}=-\frac{1}{\nu_{1}^{\mathrm{eq}}} \boldsymbol{\Lambda}-\frac{1}{\nu_{2}^{\mathrm{eq}}} \boldsymbol{\Lambda} \cdot \boldsymbol{M}^{\mathrm{eq}} \boldsymbol{M}^{\mathrm{eq}},
$$

where $\nu_{i}^{\text {eq }}$ denote the equilibrium values of the coefficients $\nu_{i}$. Decomposing the off-equilibrium magnetization into components parallel and perpendicular to the magnetic field direction, $\boldsymbol{M}=\boldsymbol{M}^{\|}+\boldsymbol{M}^{\perp}$, one finds from (53)

$$
\dot{\boldsymbol{M}}=-\frac{1}{\tau^{\perp}} \boldsymbol{M}^{\perp}-\frac{1}{\tau^{\|}}\left(\boldsymbol{M}^{\|}-\boldsymbol{M}^{\mathrm{eq}}\right),
$$

where the field-dependent relaxation times are defined by

$$
\tau^{\perp}=\frac{3 S_{1}^{\mathrm{eq}}(\alpha)}{D_{\mathrm{rot}} \alpha\left(2+S_{2}^{\mathrm{eq}}(\alpha)\right)}, \tau^{\|}=\frac{3 S_{1}^{\mathrm{eq}}(\alpha)}{2 D_{\mathrm{rot}}\left(1-S_{2}^{\mathrm{eq}}(\alpha)\right)} .
$$

Linearization in the volume fraction $\phi$ leads to

$$
\begin{aligned}
& \tau^{\perp}=\tau_{0}^{\perp}\left(1+\chi_{\mathrm{L}} t_{1}^{\perp}+\lambda^{2} \phi t_{2}^{\perp}\right), \\
& \tau^{\|}=\tau_{0}^{\|}\left(1+\chi_{\mathrm{L}} t_{1}^{\|}+\lambda^{2} \phi t_{2}^{\|}\right),
\end{aligned}
$$

where

$$
\tau_{0}^{\perp}=\frac{L_{1}(\alpha)}{D_{\operatorname{rot}}\left(\alpha-L_{1}(\alpha)\right)}, \tau_{0}^{\|}=\frac{\alpha L_{1}^{\prime}(\alpha)}{2 D_{\operatorname{rot}} L_{1}(\alpha)}
$$



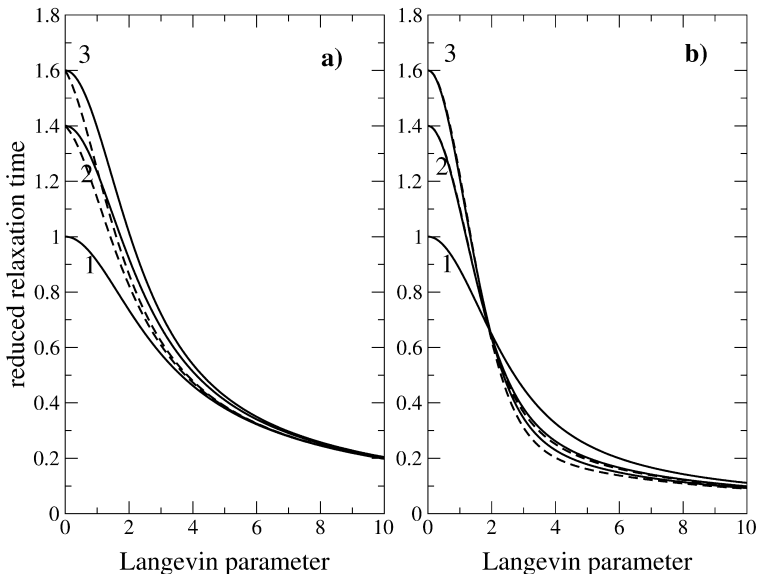

Fig. 4. a) Reduced transverse, $\tau^{\perp} / \tau_{\text {rot }}$, and b) parallel, $\tau^{\|} / \tau_{\text {rot }}$, relaxation times as functions of the applied magnetic field $\alpha$. The volume fraction was chosen as $\phi=0.15$. Curve 1 corresponds to $\lambda=0$, curve 2 to $\lambda=1$, and curve 3 to $\lambda=1.5$. Solid lines are the result of (56) and (57), dashed lines are the result of [10].

are the corresponding relaxation times in the noninteracting system and the functions $t_{i}^{\perp}$ and $t_{i}^{\|}$are defined in appendix. For the case of vanishing magnetic field, $\alpha \rightarrow 0$, the above expressions coincide, $\tau^{\perp}(0)=\tau^{\|}(0)=\tau_{\text {rot }}\left(1+\chi_{\mathrm{L}} / 3\right)$. Note, that no contribution from $\mathcal{O}\left(\lambda^{2}\right)$ remains in this limit. Thus, dipolar interactions lead to an increase of the zero-field relaxation time compared to the dilute suspension. In Fig. 4 we plot the relaxation times $\tau^{\perp}$ and $\tau^{\|}$as functions of the magnetic field $\alpha$ for $\phi=0.15$ and $\lambda=0,1,1.5$, respectively. From Fig. 4 we notice that the transverse relaxation time is enhanced due to dipolar interactions for arbitrary values of the magnetic field. As the magnetic field increases, however, the differences between $\tau^{\perp}$ and $\tau_{0}^{\perp}$ decrease. The relaxation time parallel to the magnetic field is increased compared to $\tau_{0}^{\|}$only for small magnetic fields, while it is decreased in case of strong magnetic fields. For comparison, we included in Fig. 4 also the corresponding results of [10]. Note, however, that due to the dependence of $\tau^{\perp}$ and $\tau_{0}^{\perp}$ on the coefficient $B$, the results of [10] for the relaxation times are incorrect [23].

\section{Conclusion}

In the present work we have proposed a kinetic model of dilute, weakly interacting ferrofluids that extends the classical noninteracting kinetic model of fer- rofluids [5] by the incorporation of dipolar interactions. Our model predicts several extensions compared to the classical kinetic model which are in qualitative agreement with experimental results, such as an enhanced magnetoviscous effect, modified anisotropy of the viscosity and the dependence of viscosity on the hydrodynamic volume fraction and the symmetric velocity gradient. For a quantitative comparison with experimental results, two additional parameters have to be specified which are absent in the non-interacting model [5]: the dipolar interaction parameter $\lambda$ and the ratio of the rotational over the translational relaxation time of the colloidal particles $\sigma$. While $\lambda$ is defined by (3) and also tabulated for several ferrofluids, determining $\sigma$ is not straightforward. As has been discussed above, estimations of $\sigma$ can give information only about the order of magnitude of $\sigma$, rather than a certain value. On the other hand, the value of $\sigma$ can be inferred from measurements, e.g. of the zero-shear zero-field viscosity $\eta_{0},(37)$.

\section{Acknowledgements}

This work was supported by the Deutsche Forschungsgemeinschaft (DFG) via the priority program 1104 'Colloidal magnetic fluids' under grant No. HE 1100/6-3. Valuable discussions with M. Kröger are gratefully acknowledged.

\section{Appendix A}

The functions $G_{i}\left(\alpha_{s}\right)$ are defined by $G_{i}\left(\alpha_{s}\right)=$ $G_{i}\left[f_{\text {eq }}\right]$, where the functionals $G_{i}[f]$ for $i=2,3,4$ are given by (11)-(13), and $f_{\text {eq }}$ is defined in (16). Evaluating the functionals $G_{i}[f]$ with the equilibrium distribution function $f_{\text {eq }}$ one obtains

$$
\begin{aligned}
& G_{2}(x)=\frac{4}{15}\left(L_{2}(x)^{2}+5\right), \\
& G_{3}(x)=-\frac{4}{525}\left(L_{3}(x)^{2}-21 L_{1}(x)^{2}\right), \\
& G_{4}(x)=\frac{4}{3675}\left(L_{4}(x)^{2}+20 L_{2}(x)^{2}+49\right) .
\end{aligned}
$$

The functions $G_{i}(x)$, defined in $(\mathrm{A} 1-\mathrm{A} 3)$, are monotonously increasing functions of $x$. These functions depend only weakly on $x$ and have the following expansion for $x \rightarrow 0$ and $x \rightarrow \infty$ :

$G_{2}(x)= \begin{cases}\frac{4}{3}+\frac{4}{3375} x^{4}+\mathcal{O}\left(x^{5}\right) & \text { for } x \rightarrow 0 \\ \frac{8}{5}\left(1-x^{-1}\right)+\mathcal{O}\left(x^{-2}\right) & \text { for } x \rightarrow \infty,\end{cases}$ 


$$
\begin{aligned}
& G_{3}(x)=\left\{\begin{array}{lll}
\frac{4}{225} x^{2}-\frac{8}{3375} x^{4}+\mathcal{O}\left(x^{5}\right) & \text { for } x \rightarrow 0 & t_{2}^{\perp}(x)=\frac{G_{2}^{\prime}(x)}{L_{1}(x)}-\frac{x J_{2}^{\prime}(x)}{3\left(x-L_{1}(x)\right)}, \\
\frac{16}{105}\left(1-\frac{8}{5} x^{-1}\right)+\mathcal{O}\left(x^{-2}\right) & \text { for } x \rightarrow \infty, & t_{1}^{\|}(x)=\frac{L_{1}(x)}{x}
\end{array}\right. \\
& G_{4}(x)=\left\{\begin{array}{lll}
\frac{4}{75}+\frac{16}{165375} x^{4}+\mathcal{O}\left(x^{5}\right) & \text { for } x \rightarrow 0 & +\frac{2 L_{1}(x)}{L_{1}^{\prime}(x)}\left[L_{1}(x)\left(L_{1}(x)^{2}-L_{2}(x)\right)-\frac{L_{2}(x)}{x}\right], \\
\frac{8}{105}\left(1-\frac{67}{35} x^{-1}\right)+\mathcal{O}\left(x^{-2}\right) \text { for } x \rightarrow \infty . & t_{2}^{\|}(x)=\frac{G_{2}^{\prime \prime}(x)}{L_{1}^{\prime}(x)}+\frac{x J_{2}^{\prime}(x)}{3 L_{1}(x)} .
\end{array}\right.
\end{aligned}
$$

\section{Appendix B}

The contribution of dipolar interactions to the transverse and parallel relaxation times are described by the functions

$t_{1}^{\perp}(x)=L_{1}^{\prime}(x)-\frac{L_{1}(x)}{x-L_{1}(x)}\left(L_{1}(x)^{2}-L_{2}(x)\right)$

[1] J. P. McTague, J. Chem. Phys. 51, 133 (1969).

[2] E. Blums, A. Cebers, and M. M. Maiorov, Magnetic Fluids, de Gruyter, Berlin 1997.

[3] S. Odenbach, Magnetoviscous Effects in Ferrofluids, Lecture Notes in Phys., Springer, Berlin 2002.

[4] M. Babincova, P. Babinec, and C. Bergemann, Z. Naturforsch. 56a, 909 (2001).

[5] M. A. Martsenyuk, Y. L. Raikher, and M. I. Shliomis, Sov. Phys. JETP 38, 413 (1974).

[6] S. Odenbach, Int. J. Mod. Phys. B 14, 1615 (2000).

[7] A. Y. Zubarev and L. Y. Iskakova, Phys. Rev. E 61, $5415(2000)$

[8] B. Huke and M. Lücke, Phys. Rev. E 62, 6875 (2000).

[9] G. P. Bogatyrev and V. G. Gilev, Magnetohydrodynamics 20, 249 (1984).

[10] A. Y. Zubarev and A. V. Yushkov, JETP 87, 484 (1998).

[11] S. Odenbach and H.W. Müller, Phys. Rev. Lett. 89, 037202 (2002).

[12] H.W. Müller and M. Liu, Phys. Rev. E 64, 061405 (2001).

The functions $t_{i}^{\perp}$ are positive, while the functions $t_{i}^{\|}$ are not sign-definite. In the limit $x \rightarrow \infty$ we find $t_{i}^{\perp} \rightarrow 0$ and $t_{i}^{\|} \rightarrow 0$, while for $x \rightarrow 0$ the following asymptotic behavior is obtained: $t_{1}^{\perp}(x)=1 / 3-$ $4 x^{2} / 45, t_{2}^{\perp}(x)=4 x^{2} / 375, t_{1}^{\|}(x)=1 / 3-7 x^{2} / 45$, and $t_{2}^{\|}(x)=56 x^{2} / 1125$.

[13] L. Onsager, Ann. (N. Y.) Acad. Sci. 51, 627 (1949).

[14] Z. Wang, C. Holm, and H. W. Müller, Phys. Rev. E 66, 021405 (2002).

[15] S. Hess, Z. Naturforsch. 31a, 1034 (1976).

[16] U. M. B. Marconi and P. Tarazona, J. Chem. Phys. 110, 80328044 (1999).

[17] P. Ilg and M. Kröger, Phys. Rev. E 66, 021501 (2002).

[18] N. A. Clark and B. J. Ackerson, Phys. Rev. Lett. 44, 1005 (1980).

[19] S. Hess, Phys. Rev. A 22, 2844 (1980).

[20] J. L. McWhirter and G. N. Patey, J. Chem. Phys. 117, 2747 (2002).

[21] S. Hess, in Rheological Modelling: Thermodynamic and Statistical Approaches, ed. by D. J.J. CasasVázquez, Springer, Berlin 1990, vol. 381 of Lecture Notes in Phys., pp. 51-73.

[22] P. Ilg, M. Kröger, and S. Hess, J. Chem. Phys. 116, 9078 (2002)

[23] A. Y. Zubarev, private communication (2003). 